\title{
Performance Comparison of Managed C\# and Delphi Prism in Visual Studio and Unmanaged Delphi 2009 and C++ Builder 2009 Languages
}

\author{
Abdulkadir Karaci \\ Kastamonu University \\ Department of Computer and \\ Instructional Technology Education, \\ Education Faculty, Turkey
}

\begin{abstract}
Managed C\# and Delphi Prism in Visual Studio 2008 and Unmanaged Delphi 2009 and C++ Builder 2009 programming languages are increasingly gaining in popularity. In this study, response times of these languages, memory consumptions and code lengths were tested with various work loads and the results belonging to these tests were given. Whether there was a significant difference among the data obtained by the test results was tested by using Friedman test and a significant difference was found. Also, the differences between managed languages and unmanaged languages were revealed by the results of the performance test.
\end{abstract}

\section{Keywords}

Performance Test, Programming Language, C\#, Delphi, Managed, Unmanaged.

\section{INTRODUCTION}

Today most of software developers prefer managed languages because managed languages have the properties of (1) memory and data type security, (2) automatic memory management, (3) dynamic code conduction, (4) determining the boundaries between codes having type security and not having security. Also, most of these languages are object-based [1].

The lan guages C\#, Delphi Prism in Visual Studio 2008, Java are managed languages. The languages C, C++, Delphi 2009, C++ Builder 2009 are unmanaged languages. There is not an automatic memory method in the unmanaged languages and they are not safe.

The NET platform of the Microsoft has been designed in order to develop Windows applications more easily by ensuring a sound framework [2]. The NET Framework is a complete "application" development platform, which has been developed by the Microsoft and, which has been established on open Internet protocols and standards. It bears significant resemblances to the Java Platform, which has been developed by the Sun Microsystems before. The scope of application concept here is very broad. Everything from a desktop application to a web browser has been considered within this platform and has been supported. It has been made possible that it can establish web services easily for its communication with all the applications in the world and with each other regardless of the setting in which it was developed. This platform has been designed as highly more movable than the operation system and hardware [3].

Programmers and computer scientists have been working on the advantages and disadvantages of various programming languages [4]. For the purpose of contributing to the studies in this field, in this article, the managed C\#, Delphi Prism in Visual Studio 2008 and the unmanaged Delphi 2009, C++ Builder 2009 programming languages are compared in terms of their response time, memory consumptions and code lengths. Also, the response times, memory consumptions and code lengths of the managed and unmanaged languages are compared. Thus, whether the managed lan guages, which are superior in terms of safety, are superior in terms of speed (working time - response time) will be revealed.

\section{THE EXPERIMENTAL STUDY}

Of the 400 software engineering research articles, which need experimental validation, 40 percent do not include experimental knowledge at all and this rate in other disciplines 15 percent [5]. Therefore, in this article, for the purpose of testing the performances of the programming languages, the experimental programs have been prepared and whether there is a significant difference among the experimental results obtained have been tested with the Friedman test.

\subsection{The Test Platform}

2.1.1.The Structure of the Programming Languages Compared

The properties belonging to the programming lan guages, the performances of which are compared are shown in Table 1.

Table 1. The Properties of the Programming Languages Used in the Performance Test

\begin{tabular}{|l|l|l|l|}
\hline \multicolumn{1}{|c|}{ Programming Language } & \multicolumn{1}{|c|}{ Model of execution } & \multicolumn{1}{|c|}{$\begin{array}{c}\text { Primary } \\
\text { purpose }\end{array}$} & \multicolumn{1}{c|}{$\begin{array}{c}\text { Memory } \\
\text { management }\end{array}$} \\
\hline C++ Builder 2009 & CodeGear C++ Compiler 6.10 (bcc32) [6] & Application & Manual \\
\hline Delphi 2009 & $\begin{array}{l}\text { High-performance 32-bit optimizing Delphi® native } \\
\text { code compiler [7] }\end{array}$ & Application & Manual \\
\hline C\# 3.0 & JIT compiled [8,9] & Application & Automatic \\
\hline Delphi Prism in Visual Studio 2008 & Rem Objects Oxy gen compiler & Application & Automatic \\
\hline
\end{tabular}


C\# is a powerful component oriented but a simple language of the Microsoft primarily aiming at application developers and developing applications by using the Microsoft .Net Framework. C\# plays a significant role in the Microsoft NET Framework engineering. $\mathrm{C \#}$ bears the most of the best properties of $\mathrm{C}++$ and Visual Basic; however, some of their inconsistencies and working time errors have been eliminated, as a result, a clearer and more logical lan guage has emerged $[10,11]$.

As C\#, Delphi Prism is a language working in integration with Visual Studio. Delphi Prism is preferred in order to develop desktop and web applications by using the Visual Studio and Delphi programming language. Prism, which is used in the Visual Studio platform is not $100 \%$ compatible with Delphi. However, there are additions to and developments in the Delphi Prism and Delphi.

The Delphi Prism, Delphi 2009, C++ Builder 2009 are the programming languages produced by Code Gear and contained in RAD studio. With Code Gear RAD Studio 2009, Windows.NET, Web and database applications can be developed. Delphi 2009 and C++Builder 2009 offer the fastest way to build highly performative native Windows applications. Delphi and C++Builder include visual designers and hundreds of components to easily create rich user interfaces and versatile database applications. RAD Studio's Delphi Prism, powered by the RemObjects Oxygene compiler, enables development for both .NET and mono applications, and provides support for the latest .NET
Framework technologies including ASP.NET, WinForms, WPF and LINQ [12,13].

\subsubsection{The Computer Properties}

The properties of the computer used in the test are as in the following:

- ASUS F3J series Notebook

- 100 Gigaby te Hard disk

- 2 Gigaby te RAM

- Intel Core 2 1.83 Gigahertz Processor

- Windows XP Professional Operation Sy stem

\subsection{Workloads}

The workload concept is an ultra significant component in the problem of modeling computer systems [8]. The focus of performance evaluations on workload decreases costs and quantity of simulation [14]. Experimental system evaluations generally contain a set of programs representing workload system. Every performance evaluation program is run with systems having different properties. The behavior of the system is measured and its performance is commented [15]. Workload contains a list of demands of the service from the system. For example, workload constructed in order to compare some database systems a group of queries [2]. Workloads in this study are made up of programs, each of which measures a different property of the programming language. These workloads are shown in Table 2.

Table 2. Workload

\begin{tabular}{|l|l|}
\hline Workload Code & Explanation \\
\hline Hello (1) & Printing of "Hello World" on the screen for 5000 times \\
\hline Matrix (2) & Multiplication of two matrices of 500 x 500 dimensions \\
\hline Sorting (3) & $\begin{array}{l}\text { Sorting of the series with 10000 elements, the element values of which are in the worst situation with the } \\
\text { Selection Sorting algorithm. }\end{array}$ \\
\hline Sieve (4) & Estimation of the prime numbers at the interval of [1..8193] with the sieve algorithm for 10.000 times \\
\hline Empty Loop (5) & The empty loop at the interval of [1.. 100000000] \\
\hline Mean (6) & Estimation of the mean of the numbers at the interval of [1..3000] for 30000 times \\
\hline Table (7) & $\begin{array}{l}\text { Writing and Reading of the character knowled ge } \\
\text { "abcdefghijklmnopqrstuvwxyz1234567890abcdefghijk lmnopqrstuvwxyz123456 7890abcdefgh" with a text file } \\
\text { for 10000 times }\end{array}$ \\
\hline
\end{tabular}

The algorithms used as workloads have been coded in every language, the performance of which will be tested by using standard properties in a way that they are equal to each other. These coded programs have been transformed into executable code and their memory consumptions have been obtained from Windows Operation System command prompt.

The Hello (1) program tests writing on the screen and loading performance of the program, Matrix (2) and Mean (6) programs integer arithmetic performance, Sorting (3) program loop and logical decision performance. The Sieve (4) program estimates prime numbers by using the classical Sieve Eratoshene algorithm. The Sieve program tests the basic integer arithmetic and logical comparison operation [16]. The Empty Loop (5) program tests the loop performance, Table (7) program tests writing in the text file and reading performance.

\subsection{Performance Metric}

The performance metrics used in the testing of the performance of the programming lan guages are shown in Table 3.

Table 3. Performance Metrics

\begin{tabular}{|c|l|}
\hline \multicolumn{1}{|c|}{ Performance Metrics } \\
\hline 1 & Code Len gth (LOC/CLOC) \\
\hline 2 & Response Time (ms (millisecond)) \\
\hline 3 & Memory Use (KB (Kilobyte)) \\
\hline
\end{tabular}

\subsubsection{The Code Length of the Programs Written}

The number of the code is commonly used in order to measure the source code length of a program. (LOC (line of code)) [17]. The number of line is defined as LOC=NLOCK+CLOCK. 
NLOCK (Uncommented Source Line of Code) is a code line which is not used during compilation. CLOCK (A Commented Source Line of Code) is a code line which is used during compilation. The best estimation should generally be performed as in the following in order to estimate the source code length of a program:

1. Empty lines

2. Lines involved in compilation (CLOC)
3. Data definitions and other commands

4. Lines produced by the software development instrument

The density of the lines compiled in a program can be estimated with CLOC/LOC formula [18].

The line numbers of program codes used as a workload in this study have been estimated in line with the explanations stated above and they have been shown in Table 4 .

Table 4. Code Lengths of the Programs Written

\begin{tabular}{|c|c|c|c|c|c|c|c|c|c|c|}
\hline \multirow[t]{2}{*}{ Test } & \multicolumn{5}{|c|}{ C\# } & \multicolumn{5}{|c|}{ Delphi Prism (Delphi 2009.net) } \\
\hline & CL* & $\begin{array}{c}\text { Data } \\
\text { Definitions }\end{array}$ & $\begin{array}{c}\text { Code } \\
\text { Produced by } \\
\text { the Language }\end{array}$ & $\mathbf{L}^{*}$ & $\mathbf{C L} / \mathbf{L}$ & $\mathbf{C L}^{*}$ & $\begin{array}{c}\text { Data } \\
\text { Definitions }\end{array}$ & $\begin{array}{c}\text { Code } \\
\text { Produced by } \\
\text { the Language }\end{array}$ & $\mathbf{L}^{*}$ & CL/L \\
\hline Hello (1) & 5 & 1 & 9 & 15 & $\mathbf{0 , 3 3}$ & 5 & 1 & 13 & 19 & 0,26 \\
\hline Matrix (2) & 19 & 5 & 9 & 33 & $\mathbf{0 , 5 8}$ & 17 & 3 & 13 & 33 & 0,52 \\
\hline Sort (3) & 15 & 3 & 9 & 27 & $\mathbf{0 , 5 6}$ & 16 & 3 & 13 & 28 & 0,57 \\
\hline Sieve (4) & 20 & 3 & 9 & 32 & $\overline{\mathbf{0 , 6 3}}$ & 23 & 3 & 13 & 39 & 0,59 \\
\hline Empty Loop (5) & 4 & 2 & 9 & 15 & $\mathbf{0 , 2 7}$ & 4 & 2 & 13 & 19 & 0,21 \\
\hline Mean (6) & 9 & 2 & 9 & 20 & $\mathbf{0 , 4 5}$ & 9 & 3 & 13 & 25 & 0,36 \\
\hline Table (7) & 18 & 8 & 9 & 35 & $\mathbf{0 , 5 1}$ & 21 & 5 & 13 & 39 & 0,54 \\
\hline Mean & & & & & 0,48 & & & & & 0,44 \\
\hline Test & \multicolumn{5}{|c|}{ Delphi 2009} & \multicolumn{5}{|c|}{ C Builder 2009} \\
\hline & CL* & $\begin{array}{c}\text { Data } \\
\text { Definitions }\end{array}$ & $\begin{array}{c}\text { Code } \\
\text { Produced by } \\
\text { the Language }\end{array}$ & $\mathbf{L}^{*}$ & $\mathbf{C L} / \mathbf{L}$ & CL* & $\begin{array}{c}\text { Data } \\
\text { Definitions }\end{array}$ & $\begin{array}{c}\text { Code } \\
\text { Produced by } \\
\text { the Language }\end{array}$ & $\mathbf{L}^{*}$ & CL/L \\
\hline Hello (1) & 4 & 2 & 5 & 11 & 0,36 & 5 & 3 & 3 & 11 & 0,45 \\
\hline Matrix (2) & 17 & 3 & 5 & 25 & 0,68 & 19 & 6 & 3 & 28 & $\mathbf{0 , 6 8}$ \\
\hline Sort (3) & 13 & 3 & 5 & 21 & 0,62 & 16 & 4 & 3 & 23 & 0,70 \\
\hline Sieve (4) & 28 & 3 & 5 & 36 & $\overline{\mathbf{0 , 7 8}}$ & 23 & 10 & 3 & 36 & 0,64 \\
\hline Empty Loop (5) & 4 & 3 & 5 & 12 & $\mathbf{0 , 3 3}$ & 5 & 3 & 3 & 11 & 0,45 \\
\hline Mean (6) & 8 & 3 & 5 & 16 & $\mathbf{0 , 5 0}$ & 9 & 5 & 3 & 17 & $\mathbf{0 , 5 3}$ \\
\hline Table (7) & 21 & 4 & 5 & 30 & $\mathbf{0 , 7 0}$ & 23 & 7 & 3 & 33 & $\mathbf{0 , 7 0}$ \\
\hline Mean & & & & & $\mathbf{0 , 5 7}$ & & & & & 0,59 \\
\hline
\end{tabular}

* L:LOC CL:CLOC

The graphic of CLOC/LOC values given in Table 4 are shown in Figure 1.

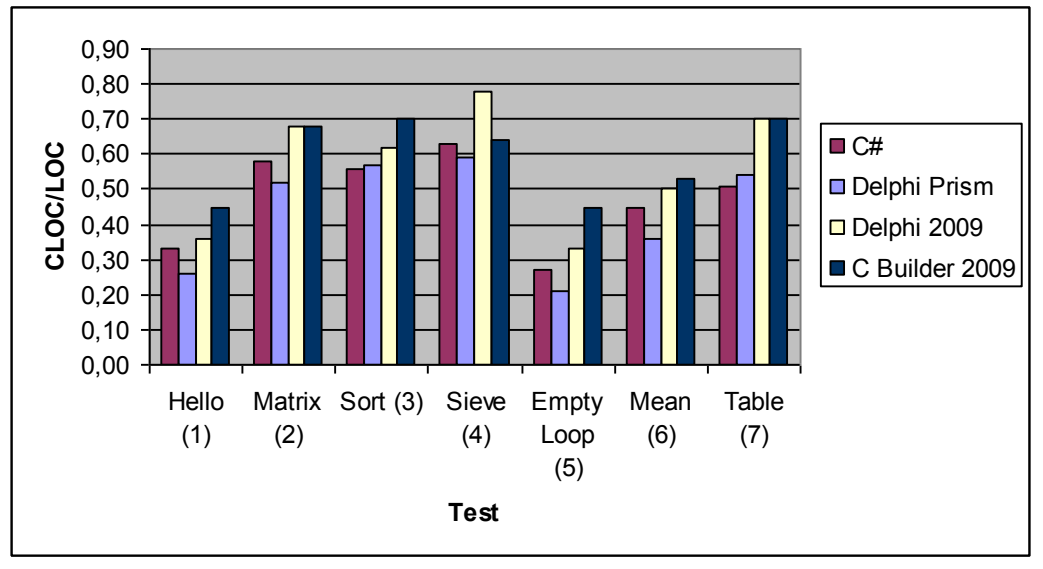

Fig 1: Code density graphic compiled (CLOC/LOC) 


\subsubsection{Response Time}

Response time is a significant concept in computer systems performance studies. Response time is the measurement of the time for which a user or an application has to wait until a command requested is completed [8]. In this study, response times of workloads run in the programming lan guages desired to be measured are given in Table 5 .

Table 5. Response Time of Workloads on Windows Operation System (ms millisecond)

\begin{tabular}{|c|c|c|c|c|c|c|c|c|c|c|c|c|}
\hline Test & Min & Max & Mean & Min & $\overline{\mathrm{Max}}$ & Mean & Min & Max & Mean & Min & $\overline{\mathrm{Max}}$ & $\overline{\overline{\text { Mean }}}$ \\
\hline Matrix (2) & 3281 & 3562 & 3397,95 & 3062 & 3328 & 3168,05 & 953 & 1172 & 1076,5 & 515 & 625 & $\underline{554,2}$ \\
\hline Sort (3) & 343 & 453 & 389,05 & 500 & 609 & 548,2 & 578 & 703 & 653,15 & 109 & 204 & $\underline{175,1}$ \\
\hline Sieve (4) & 1546 & 1640 & 1596,25 & 1468 & 1515 & 1497,1 & 6797 & 6860 & 6824,225 & 766 & 844 & $\underline{791,425}$ \\
\hline $\operatorname{Mean}(6)$ & 203 & 343 & 246,25 & 312 & 375 & 338,575 & 437 & 500 & 451,875 & 78 & 94 & $\underline{91,775}$ \\
\hline Table(7) & 15 & 62 & $\underline{\underline{25,775}}$ & 93 & 156 & 110,6 & 343 & 422 & 355,425 & 62 & 79 & 72,325 \\
\hline Mean & & & 891.79 & & & 875.39 & & & 1450.46 & & & $\underline{300.46}$ \\
\hline
\end{tabular}

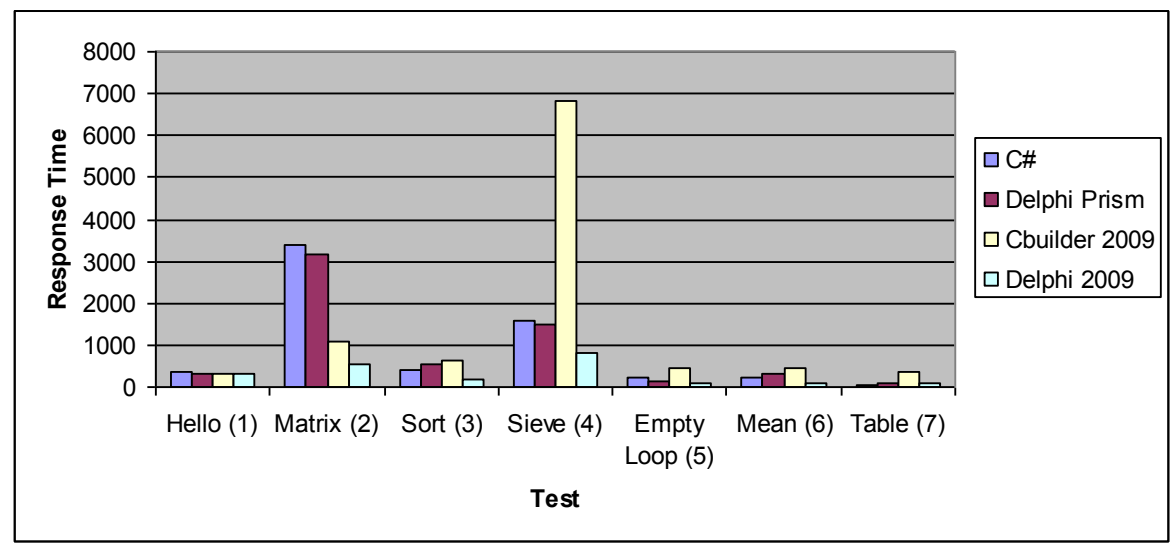

Fig 2: Response Time Graphic of Workloads on Windows Operation System

Mean response times of all workloads by programming lan guages are shown in Figure 3.

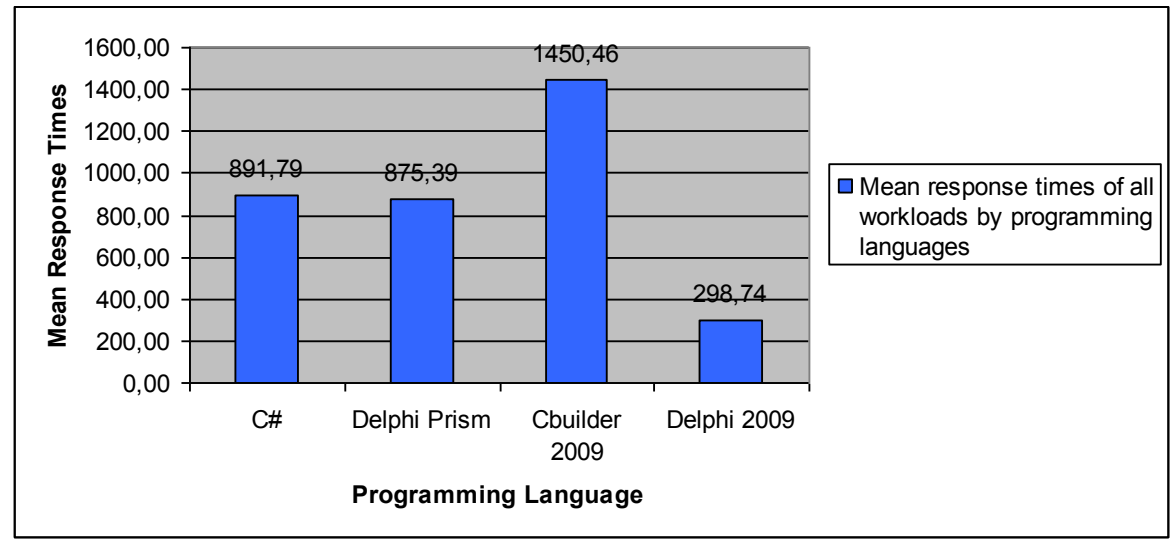

Fig 3: Mean Response Times of All the Workloads by the Programming Lan guages 


\subsubsection{Memory Consumption}

Memory consumption of every workload has been obtained separately by programming languages by using Memory Booster Gold. These values are shown as Kilobyte (KB) in Table 6.

Table 6. Memory Consumption (KB)

\begin{tabular}{|l|c|c|c|c|}
\hline \multicolumn{1}{|c|}{ Workload } & C\# & $\begin{array}{c}\text { Delphi Prism } \\
\text { (Del phi 2009.net) }\end{array}$ & C++ Builder 2009 & Delphi 2009 \\
\hline Hello (1) & 4404 & 4796 & 3828 & 1280 \\
\hline Matrix (2) & 6648 & 7352 & 3516 & 3312 \\
\hline Sort (3) & 4620 & 4572 & 1328 & 3828 \\
\hline Sie ve (4) & 4280 & 4568 & 1308 & 1416 \\
\hline Empty Loop (5) & 4100 & 4476 & 1288 & 1336 \\
\hline Mean (6) & 4340 & 4508 & 1316 & 1336 \\
\hline Table (7) & 14656 & 9654 & 1420 & 1388 \\
\hline Mean & 6149,71 & 5703,71 & 2000,57 & $\mathbf{1 9 8 5 , 1 4}$ \\
\hline
\end{tabular}

The graphic belonging to memory consumption data is shown in Figure 4.

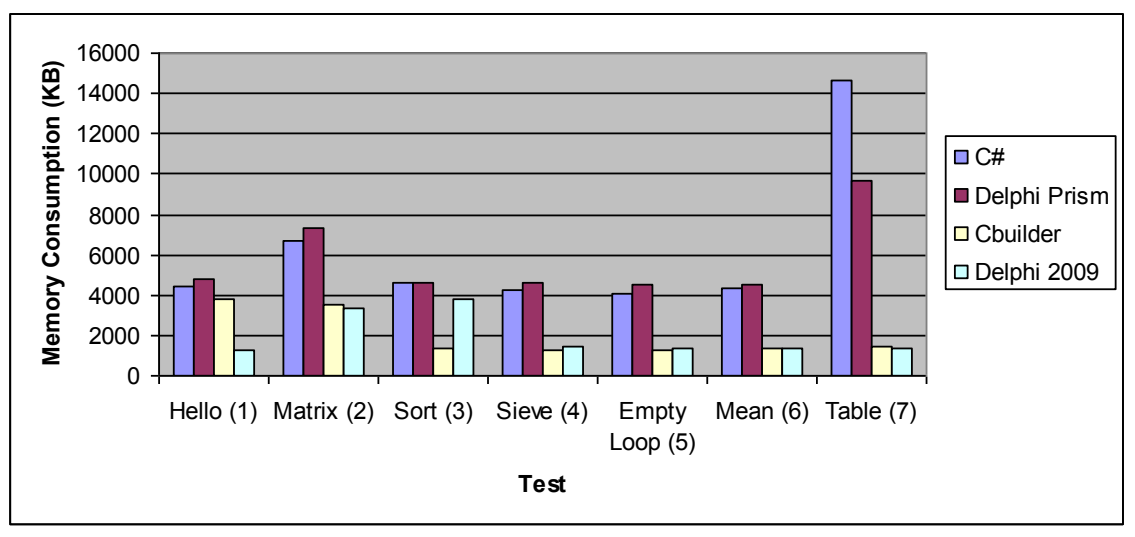

Fig 4: Memory Consumption Graphic

\subsection{Statistical Design}

Minimal descriptive statistics contains the following for a data set: total observation number, mean, median, standard deviation, minimal value, maximum value and number of observations Presentation of descriptive statistics data on dependent variable is significant [19]. Therefore, descriptive statistics data obtained are shown in detail by programming languages in Table 7 .

Table 7. Descriptive Statistics

\begin{tabular}{|c|c|c|c|c|c|c|}
\hline \multicolumn{7}{|c|}{ Dependent Variable: Response Time } \\
\hline Workload & $\begin{array}{l}\text { Programming } \\
\text { Language }\end{array}$ & Mean & Std. De viation & Sub Limit & Upper Limit & $\mathbf{N}$ \\
\hline \multirow{5}{*}{ Hello [1] } & $\mathrm{c \#}$ & 342,5250 & 29,80362 & 332,616 & 352,434 & 40 \\
\hline & d2009net & 318,7250 & 17,41645 & 308,816 & 328,634 & 40 \\
\hline & $\mathrm{d} 2009$ & 317,6750 & 15,98459 & 307,766 & 327,584 & 40 \\
\hline & cbuilder & 334,4500 & 14,42034 & 324,541 & 344,359 & 40 \\
\hline & Total & & & & & 160 \\
\hline \multirow{5}{*}{ Matrix [2] } & $\mathrm{c \#}$ & 3397,9500 & 96,45564 & 3388,041 & 3407,859 & 40 \\
\hline & d2009net & 3168,0500 & 93,90174 & 3158,141 & 3177,959 & 40 \\
\hline & $\mathrm{d} 2009$ & 554,2000 & 22,09444 & 544,291 & 564,109 & 40 \\
\hline & cbuilder & 1076,5000 & 50,49295 & 1066,591 & 1086,409 & 40 \\
\hline & Total & & & & & 160 \\
\hline \multirow{5}{*}{ Sort [3] } & $\mathrm{c \#}$ & 389,0500 & 19,13776 & 379,141 & 398,959 & 40 \\
\hline & d2009net & 548,2000 & 21,57777 & 538,291 & 558,109 & 40 \\
\hline & $\mathrm{d} 2009$ & 175,1000 & 25,53610 & 165,191 & 185,009 & 40 \\
\hline & cbuilder & 653,1500 & 23,80428 & 643,241 & 663,059 & 40 \\
\hline & Total & & & & & 160 \\
\hline \multirow{2}{*}{ Sieve [4] } & $\mathrm{c \#}$ & 1596,2500 & 13,40254 & 1586,341 & 1606,159 & 40 \\
\hline & d2009net & 1497,1000 & 9,98922 & 1487,191 & 1507,009 & 40 \\
\hline
\end{tabular}




\begin{tabular}{|c|c|c|c|c|c|c|}
\hline & $\mathrm{d} 2009$ & 791,4250 & 18,17703 & 781,516 & 801,334 & 40 \\
\hline & cbuilder & 6824,2250 & 13,60522 & 6814,316 & 6834,134 & 40 \\
\hline & Total & & & & & 160 \\
\hline \multirow{5}{*}{ Empty [5] } & $\mathrm{c \#}$ & 244,7 & 15,92804 & 234,791 & 254,609 & 40 \\
\hline & d2009net & 146,475 & 17,25523 & 136,566 & 156,384 & 40 \\
\hline & $\mathrm{d} 2009$ & 100,75 & 7,87645 & 90,841 & 110,659 & 40 \\
\hline & cbuilder & 457,625 & 22,59077 & 447,716 & 467,534 & 40 \\
\hline & Total & & & & & 160 \\
\hline \multirow{5}{*}{ Mean [6] } & $\mathrm{c \#}$ & 246,25 & 23,58482 & 236,341 & 256,159 & 40 \\
\hline & d2009net & 338,575 & 11,74054 & 328,666 & 348,484 & 40 \\
\hline & $\mathrm{d} 2009$ & 91,775 & 5,21579 & 81,866 & 101,684 & 40 \\
\hline & cbuilder & 451,875 & 15,60603 & 441,966 & 461,784 & 40 \\
\hline & Total & & & & & 160 \\
\hline \multirow{5}{*}{ Table [7] } & $\mathrm{c \#}$ & 25,775 & 9,75202 & 15,866 & 35,684 & 40 \\
\hline & d2009net & 110,6 & 18,17127 & 100,691 & 120,509 & 40 \\
\hline & d2009 & 72,325 & 7,69411 & 62,416 & 82,234 & 40 \\
\hline & cbuilder & 355,425 & 18,26849 & 345,516 & 365,334 & 40 \\
\hline & Total & & & & & 160 \\
\hline \multirow{5}{*}{ Total } & $\mathrm{c \#}$ & 891,7857 & 1131,39129 & & & 280 \\
\hline & d2009net & 875,3893 & 1035,17521 & & & 280 \\
\hline & $\mathrm{d} 2009$ & 300,4643 & 256,45752 & & & 280 \\
\hline & cbuilder & 1450,4643 & 2210,59212 & & & 280 \\
\hline & Total & & & & & 1120 \\
\hline
\end{tabular}

ANOVA is used when searching the effect of two independent variables on a dependent variable [20]. In this study, the dependent variable is response time, the independent variables are the program and the programming languages. Significant results have been obtained by applying two-way ANOVA on these dependent and independent variables. However, because variance equality assumption has not been ensured, these results have not been presented in the article. Instead, the Friedman test, a non-parametric method, has been used.

The Friedman test is the non-parametric correspondence of twoway ANOVA test. When the same samples belonging to the subjects have been treated and when these samples have been measured at three or more points, the Friedman test is used $[20,21]$.
The Friedman test has been used in order to find whether there is a significant difference among response times obtained as a result of running of every workload on C\#, Delphi Prism, Delphi 2009 and C Builder 2009 programming languages. A significant difference has been found among response times obtained from 4 different programming languages as a result of the analysis of response times [ $\chi^{2}(\mathrm{df}=3, \mathrm{~N}=280)=486.261, \mathrm{p}<.05$ ] obtained as a result of running of workloads on programming lan guages. The test data obtained from the Friedman test is shown in Table 8.

Table 8. The Friedman Test Data

\begin{tabular}{|c|c|c|c|c|c|c|c|}
\hline $\begin{array}{l}\text { The Programming } \\
\text { Lan guage }\end{array}$ & $\mathrm{N}$ & Mean & Std. Deviation & Mean Rank & $\chi^{2}$ & df & $\mathrm{P}$ \\
\hline $\mathrm{C} \#$ & 280 & 891.7857 & 1131,39129 & 2.61 & \multirow{4}{*}{486.261} & \multirow{4}{*}{3} & \multirow{4}{*}{.000} \\
\hline Delphi Prism & 280 & 875.3893 & 1035,17521 & 2.51 & & & \\
\hline Delphi 2009 & 280 & 300.4643 & 256,45752 & 1.25 & & & \\
\hline C Builder 2009 & 280 & 1450.4643 & 2210,59212 & 3.63 & & & \\
\hline
\end{tabular}

\section{RESULTS AND DISCUSSION}

When the general means of response times belonging to all the workloads obtained from performance tests, the Delphi 2009 programming language is in average three times as fast as $\mathrm{C \#}$ and Delphi Prism languages and five times as fast as $\mathrm{CH}$ Builder 2009 languages. When C\# and Delphi Prism languages having Net technology are compared, Delphi Prism is $0.01 \%$ faster in terms of response time. However, because measurements have been performed on millisecond, this difference is not very significant. Also, the response times of these languages having Net technology are in average 1.6 times as fast as C++ Builder 2009 lan guage.
When response times of the programming languages are compared in detail by workloads, in all the languages, the performance of which has been tested, the loading and print on the screen speed of the programs is equal to each other with small differences which can be ignored, by the result of Hello (1) test.

By the result of Matrix (2) and Mean (6) tests, the Delphi 2009 language is approximately 5 times as fast as the C\# and Delphi Prism, and 3.5 times as fast as the $\mathrm{C}++$ Builder 2009 languages. As a result of this, the Delphi 2009 language gives a result five times as fast as the $\mathrm{C \#}$ and Delphi Prism languages, and 3.5 times as fast as the $\mathrm{C}+$ - Builder 2009 language in the integer arithmetic. 
In the logical performance by the Sort (4) test results, the Delphi 2009 is 2.2 times as fast as C\#, 3.1 times as fast as the Delphi Prism, 3.7 times as fast as the C++ Builder 2009; the Delphi Prism is 1.4 times as fast as the C\#. By the Sieve (4) test results which tested the basic integer arithmetic and logical comparison operation, the Delphi 2009 is 2 times as fast as the C\#, 1.8 times as fast as the Delphi Prism, and 8.6 times as fast as the $\mathrm{CH}$ Builder 2009. By the results of Empty (5) test which measured the loop performance, the Delphi 2009 is 2.4 times as fast as the C\#, 1.4 times as fast as the Delphi Prism, and 4.5 times as fast as the C++ Builder 2009.

Another salient result by the test results is that writing in and reading the text file speed of the $\mathrm{C \#}$ programming language is faster than the other languages. By the results of the Table (7) test, the C\# language is 2.8 times as fast as the Delphi 2009, 4.4 times as fast as the Delphi Prism, and 14.2 times as fast as the $\mathrm{C}++$ Builder 2009. Although the $\mathrm{C \#}$ language is slower than the Delphi 2009 in other tests, it is faster in writing in and reading the text file operation.

When the density of the lines compiled in the programming lan guages (CLOC/LOC), the least code density is in the Delphi Prism 2009 lan guage with a 0.44 code density mean. The code density mean of the other languages is respectively the $\mathrm{C \#} 0.48$, the Delphi 2009 0.57, and the C++ Builder 2009 0.59. The C\# and Delphi Prism languages having Net technology have less code density .

By the memory consumption mean of all the workloads, the Delphi 2009 is the least memory consuming language with a 1985.14 KB. The memory consumption mean of the other languages is respectively the $\mathrm{C}++$ Builder $2000.57 \mathrm{~KB}$, the Delphi Prism 5703.71 KB, and the C\# 6149.71 KB. The programming language, the memory consumption of which is the most is the C\#. The C\# and Delphi Prism languages consume 3 times as much memory as the other languages in average.

\section{CONCLUSION}

In terms of response time, the fastest programming language is the Delphi 2009 and the slowest programming lan guage is the $\mathrm{C}++$ Builder 2009. Although the managed language C\# and the Delphi Prism are powerful in terms of code density, they are weak in terms of memory consumption and response time.

The Delphi 2009 is the most powerful programming language both in terms of memory consumption and response time.

\section{REFERENCES}

[1] Blackburn, Stephen M.; McKinley, Kathryn S.,e.g., Wake Up and Smell the Coffee : Evaluation Methodology for the 21st Century, Communications of the ACM 51, 83-89, (2008).

[2] J.R. Dick, K. B. Kent, J. C. Libby, A quantitative analy sis of the .NET common language runtime, Journal of Systems Architecture 54, 679-696, (2008).

[3] Wikipedi World Wide Web site, http://tr.wikipedia.org/wiki/.NET_Framework

[4] ] L. Prechelt, ,An empirical comparison of seven programming languages ,Computer, 33,23 - 29, (2000).
[5] N. Juristo, A. Moreno, Basics of Software Engineering Experimentation, Kluwer Academic South America, Boston, (2001).

[6] Codegear C++ Builder (2009): C++ Builder $2009 \mathrm{Web}$ Site: http://www.codegear.com/article/38534/images/38534/CBu ilder2009FeatureM atrix.pdf

[7] Codegear Delphi (2009): Delphi 2009 Website: http://www.codegear.com/article/38548/images/38548/Del phi2009FeatureM atrix.pdf

[8] P. Fortier, H. Michel, Computer Systems Performance Evaluation and Prediction, Digital Pres,USA, Burlington, (2003) .

[9] J. G. Allen, , J. S. Jin, , Code Generation for Just-in-Time Compiled Mobile Collector Agents, ACM International Conference Proceeding Series 161,1-4, (2003).

[10] J. Sharp, Microsoft Visual C\# 2008 Step by Step, Microsoft Pres (USA, Washington, 2007).

[11] J. Sharp, Microsoft Visual C\# 2005 Step by Step, Microsoft Pres USA, Washington, (2005).

[12] CodeGear RAD Studio 2009 (2009): CodeGear RAD Studio 2009 Web Site: http://www.codegear.com/products/radstudio

[13] M. Cantù, Delphi 2007 Handbook, Wintech Italia Srl, (2007).

[14] T. M. Conte, W. Hwu, Benchmark Characterization, System Sciences, 1991. Proceedings of the Twenty-Fourth Annual Hawaii International Conference on 1 364-372, (1991).

[15] T. M. Conte, W. Hwu , "Benchmark Characterization for Experimental System Evaluation," Proc. Hawaii Int'l Conf. System Science I, 6-18, (1990).

[16] R. C. Morin, Managed C\# versus Unmanaged C++ (2009): Web Site: http://www.csharphelp.com/archives2/archive458.html

[17] M. ŞAHIN, Java, Python Ve Ruby Dillerinin Performans Karşılaştırması, Akademik Bilişimim 2007 Dumlupınar Üniversitesi, (2007).

[18] M.Chiş, Evolutionary Decision Trees and Software Metrics for Module Defects Identification, Proceedings Of World Academy Of Science, Engineering And Technology, 28, 273-277, (2008).

[19] K. El Emam, A Methodology for Validating Software Product Metrics, National Research Council of Canada,Ottawa, Ontario, Canada NCR/ERC-1076, (2000).

[20] Ş. Kalaycı,"SPSS Uygulamalı Çok Değişkenli İstatistik Teknikleri”, Asil Yayın Dağtım Turkey, Ankara, (2008).

[21] J.M. Sá, “Applied Statistics Using SPSS, STATISTICA, MATLAB and R" , Springer Berlin Heidelberg,USA, Newy ork, (2007). 